\title{
Translational Relevance of Mouse Models of Atopic Dermatitis
}

\author{
Justin Choi ${ }^{1,2}$, Nishadh Sutaria ${ }^{1}$, Youkyung Sophie Roh ${ }^{1}$, Zachary Bordeaux ${ }^{1}$, Martin P. Alphonse ${ }^{1}$, \\ Shawn G. Kwatra ${ }^{1, *}$ (i) and Madan M. Kwatra ${ }^{2}$ \\ 1 Department of Dermatology, Johns Hopkins University School of Medicine, Baltimore, MD 21231, USA; \\ justin.choi@duke.edu (J.C.); nsutari1@jhmi.edu (N.S.); sophieroh@jhmi.edu (Y.S.R.); \\ zbordeaux1@pride.hofstra.edu (Z.B.); malphon1@jhmi.edu (M.P.A.) \\ 2 Department of Anesthesiology, Duke University School of Medicine, Durham, NC 27710, USA; \\ madan.kwatra@duke.edu \\ * Correspondence: skwatra1@jhmi.edu; Tel.: +1-410-955-8662
}

Citation: Choi, J.; Sutaria, N.; Roh, Y.S.; Bordeaux, Z.; Alphonse, M.P.; Kwatra, S.G.; Kwatra, M.M. Translational Relevance of Mouse Models of Atopic Dermatitis. J. Clin. Med. 2021, 10, 613. https://doi.org/ $10.3390 / \mathrm{jcm} 10040613$

Academic Editor: Masutaka Furue Received: 16 December 2020

Accepted: 3 February 2021

Published: 6 February 2021

Publisher's Note: MDPI stays neutral with regard to jurisdictional claims in published maps and institutional affiliations.

Copyright: (C) 2021 by the authors Licensee MDPI, Basel, Switzerland. This article is an open access article distributed under the terms and conditions of the Creative Commons Attribution (CC BY) license (https:// creativecommons.org/licenses/by/ $4.0 /)$.

\begin{abstract}
The complexity of atopic dermatitis (AD) continues to present a challenge in the appropriate selection of a mouse model because no single murine model completely recapitulates all aspects of human AD. This has been further complicated by recent evidence of the distinct AD endotypes that are dictated by unique patterns of inflammation involving Th1, Th2, Th17, and Th22 axes. A review of currently used mouse models demonstrates that while all AD mouse models consistently exhibit Th2 inflammation, only some demonstrate concomitant Th17 and/or Th22 induction. As the current understanding of the pathogenic contributions of these unique endotypes and their potential therapeutic roles expands, ongoing efforts to maximize a given mouse model's homology with human $\mathrm{AD}$ necessitates a close evaluation of its distinct immunological signature.
\end{abstract}

Keywords: atopic dermatitis; eczema; mouse models; dermatology; immunology

\section{Introduction}

Atopic dermatitis (AD) is a common, relapsing inflammatory skin condition characterized by pruritic, erythematous plaques and papules typically affecting the body's flexural surfaces. While AD is known to emerge due to barrier dysfunction, aberrant immune activation, and genetic predisposition, a clear understanding of the pathogenesis of its varying clinical presentations remains under investigation. Current knowledge of AD's multifaceted pathogenesis has been predicated on a diverse array of murine models that have played a pivotal role in delineating the functions of various susceptibility genes and exogenous triggers in the disease process.

However, the heterogeneity of AD disease in humans continues to present a challenge in selecting an appropriate mouse model for preclinical studies, given that no single model fully recapitulates all aspects of human AD. This has been further complicated by the recent identification of immunologically distinct human AD subtypes that occur due to differential inflammatory axis activation [1]. As the roles of these unique inflammatory patterns and their potential therapeutic implications in AD are further clarified, the selection of appropriate mouse models based on downstream immune pathways that modulate these clinically distinct subtypes is especially important in drug validation studies.

Thus, this review seeks to evaluate commonly used mouse models for AD and to highlight the immune pathways that are affected in mice. We hope to aid investigators in the selection of appropriate models that carefully balance immune factors alongside the underlying genetics, phenotype, and transcriptomic similarities to human AD in order to optimize their translational relevance in future studies.

\section{An Overview of Mouse Models for Atopic Dermatitis}

The current repository of AD murine models reflects a broad range of mechanisms used to induce eczematous dermatitis, including the use of exogenous agents, transgenic 
mice, and inbred mice. Several of these mechanisms, such as mucosa-associated lymphoid tissue lymphoma translocation protein 1 (MALT1) deficiency, fibroblast-specific inhibitor of nuclear factor kappa-beta subunit beta (Ikk2) deficiency, and Matt deficiency, have been only loosely correlated to human $\mathrm{AD}$, and a clear understanding of their pathogenic contributions resulting in AD has yet to be fully delineated [2-4]. Nevertheless, the cutaneous inflammation observed in most models demonstrates significant overlap with key features found in human AD lesions, including elevated serum IgE, inflammatory infiltrate consisting of eosinophils, mast cells, and lymphocytes, increased epidermal thickness, hyperkeratosis, parakeratosis, acanthosis, and spongiosis [4-6].

More recently, transcriptomic analyses have measured similarities between highly differentially expressed genes in human AD and select murine models using the Metaanalysis derived atopic dermatitis transcriptome (MADAD), with data demonstrating most significant overlap with Adam $17^{\mathrm{fl} / \mathrm{fl}}$ Sox 9 Cre mice ( $34 \%$ overlap) and mice induced with IL-23 (36\% overlap) [6-8]. This is followed by NC/Nga mice, demonstrating $18 \%$ overlap with the human AD transcriptome, and oxazolone-sensitized mice, with $17 \%$ overlap [7]. Similarly, gene set enrichment analysis conducted by Nunomura et al. (2019) on Ikk2deficient (Ikk2 ${ }^{\triangle N E S}$ ) mice demonstrated a high degree of concordance with human $\mathrm{AD}$ in both upregulated (16 of 30) and downregulated (19 of 30) genes [3].

Consistent with the Th2 induction that broadly underscores all human AD endotypes, murine models invariably demonstrate Th2-biased immune response, with elevated levels of Th2-related cytokines: IL-4, IL-5, IL-13, and/or thymic stromal lymphopoietin (TSLP). Eight models (models 1-8) reported exclusively Th2 elevations, while two models (models 9-10) reported Th1 in addition to Th2 activation (Table 1). Four models (models 11-14) reported heightened Th2 and Th17 inflammation, three of which (models 12-14) also reported an increase in Th1-related cytokines (Table 2).

Table 1. Mouse models with Th2 and/or Th1 upregulation.

\begin{tabular}{|c|c|c|c|c|c|}
\hline Model & Mechanism & Features & Immune Profile & $\begin{array}{l}\text { Comparison to Human AD } \\
\text { (If Applicable) }\end{array}$ & References \\
\hline $\begin{array}{l}\text { (1) Stat6VT } \\
\text { transgenic Mice }\end{array}$ & $\begin{array}{l}\text { Transcriptionally active } \\
\text { STAT6 downregulates } \\
\text { loricrin and involucrin } \\
\text { expression in the epidermis. }\end{array}$ & $\begin{array}{l}\text { Hyperkeratosis, epidermal } \\
\text { and dermal thickening, } \\
\text { lymphocytic and } \\
\text { eosinophilic infiltrate. }\end{array}$ & Th2 (IL-4) & $\begin{array}{l}\text { Certain STAT6 intron SNPS } \\
\text { with increased promoter } \\
\text { activity were found to be } \\
\text { associated with an increased } \\
\text { risk of childhood AD. }\end{array}$ & [9-11] \\
\hline $\begin{array}{l}\text { (2) K5-tTA-IL-13 } \\
\text { mice }\end{array}$ & $\begin{array}{l}\text { Transgenic mice with } \\
\text { expression of IL-13 in the } \\
\text { skin induced with the } \\
\text { absence of a tetracycline }\end{array}$ & $\begin{array}{l}\text { Dermal and epidermal } \\
\text { thickening, spongiosis, } \\
\text { hyperkeratosis, } \\
\text { mononuclear and } \\
\text { eosinophilic infiltration }\end{array}$ & $\begin{array}{l}\text { Th2 (IL-13, } \\
\text { TSLP) }\end{array}$ & & {$[12]$} \\
\hline (3) K5-TSLP & $\begin{array}{l}\text { Tetracycline-inducible, } \\
\text { skin-specific transgene } \\
\text { expressing TSLP }\end{array}$ & $\begin{array}{l}\text { Acanthosis, spongiosis, } \\
\text { hyperkeratosis, dermal } \\
\text { mononuclear infiltrate }\end{array}$ & Th2 (IL-4, IL-5) & & [13] \\
\hline (4) hK14mIL33tg & $\begin{array}{l}\text { Transgenic mice with } \\
\text { skin-specific expression of } \\
\text { IL-33 driven by the human } \\
\text { keratin } 14 \text { promoter. }\end{array}$ & $\begin{array}{l}\text { Dermatitis with infiltrations } \\
\text { of mast cells and } \\
\text { eosinophils; increased IgE. }\end{array}$ & Th2 (IL-5, IL-13) & & [14] \\
\hline $\begin{array}{l}\text { (5) } \text { Card } 11^{\text {unm }} \\
\text { mice }\end{array}$ & $\begin{array}{l}\text { Mice harbor Card11 single } \\
\text { nucleotide variant that } \\
\text { attenuates TCR/CD28 } \\
\text { signaling to reduce the } \\
\text { efficiency of NFKB } \\
\text { signaling, resulting in the } \\
\text { gradual expansion of } \\
\text { Th2 cells. }\end{array}$ & $\begin{array}{c}\text { Acanthosis, parakeratosis, } \\
\text { and hyperkeratotic scale, } \\
\text { and heavy infiltration by } \\
\text { mast cells. }\end{array}$ & Th2 & $\begin{array}{l}\text { Dermatitis-like symptoms } \\
\text { reported in humans with } \\
\text { Card11 deficiency (Demeyer } \\
\text { et al., 2019) }\end{array}$ & [15] \\
\hline $\begin{array}{l}\text { (6) Unmodulated } \\
\text { mice with a } \\
\text { mutation in } \\
\text { Carma1/Card11 }\end{array}$ & $\begin{array}{l}\text { Mice with a mutation in } \\
\text { Carma1/Card11 have } \\
\text { impaired NFkB and } \\
\text { JNK activation. }\end{array}$ & $\begin{array}{l}\text { High serum IgE, } \\
\text { hyperkeratosis, } \\
\text { predominantly mast } \\
\text { cell infiltrate. }\end{array}$ & Th2 & $\begin{array}{l}\text { Dermatitis-like symptoms } \\
\text { reported in humans with } \\
\text { Card11 deficiency (Demeyer } \\
\text { et al., 2019) }\end{array}$ & [16] \\
\hline
\end{tabular}


Table 1. Cont.

\begin{tabular}{|c|c|c|c|c|c|}
\hline Model & Mechanism & Features & Immune Profile & $\begin{array}{l}\text { Comparison to Human AD } \\
\text { (If Applicable) }\end{array}$ & References \\
\hline $\begin{array}{l}\text { (7) Bioengineered } \\
\text { mouse model }\end{array}$ & $\begin{array}{l}\text { Bioengineered human skin } \\
\text { equivalents grafted onto } \\
\text { immunodeficient mice; } \\
\text { intradermal injection of Th2 } \\
\text { lymphocytes induce } \\
\text { AD phenotype. }\end{array}$ & $\begin{array}{l}\text { Epidermal thickening, } \\
\text { dermal angiogenic } \\
\text { response. }\end{array}$ & Th2 (TSLP) & $\begin{array}{c}\text { Allows for selective } \\
\text { introduction of specific } \\
\text { cytokines and lymphocyte } \\
\text { subsets to replicate specific } \\
\text { inflammatory patterns. } \\
\text { Potential interference with } \\
\text { mouse cytokines and } \\
\text { immune cell populations }\end{array}$ & {$[17,18]$} \\
\hline $\begin{array}{l}\text { (8) Diet-induced } \\
\text { A }\end{array}$ & $\begin{array}{l}\text { Dietary deficiency of } \\
\text { unsaturated fatty acids may } \\
\text { impair the skin } \\
\text { barrier function }\end{array}$ & $\begin{array}{l}\text { Epidermal thickening, mast } \\
\text { cell and eosinophilic } \\
\text { infiltrate, increased IgE }\end{array}$ & Th2 (IL-5, IL-13) & & [19] \\
\hline $\begin{array}{l}\text { (9) } \mathrm{EF} 1 \alpha \\
\text { transgenic mice }\end{array}$ & $\begin{array}{l}\text { Transgenic mice are driven } \\
\text { by the promoter E } \mu \text {-Lck } \\
\text { overexpressing IL-31 }\end{array}$ & $\begin{array}{c}\text { Increased pruritus, } \\
\text { hyperkeratosis, acanthosis, } \\
\text { mast cell proliferation, and } \\
\text { inflammatory infiltrate }\end{array}$ & Th1 and Th2 & & [20] \\
\hline $\begin{array}{c}(10) \\
J \mathrm{Jak}^{\text {spade/spade }}\end{array}$ & $\begin{array}{l}\text { A missense mutation in } \\
\text { JAK1, resulting in } \\
\text { hyperactivation. }\end{array}$ & $\begin{array}{l}\text { Epidermal hyperplasia, } \\
\text { mast cell/eosinophilic/ } \\
\text { lymphocytic infiltrate. }\end{array}$ & $\begin{array}{l}\text { Th2 (IL-4, IL-5, } \\
\text { IL-13) } \\
\text { earlyTh1/Th2 } \\
\text { (IFN- } \gamma \text { ) late. }\end{array}$ & & [21] \\
\hline
\end{tabular}

AD: atopic dermatitis. TSLP: thymic stromal lymphopoietin. STAT6: signal transducer and activator of transcription 6. SNP: single nucleotide polymorphism. NFKB: nuclear factor kappa B. JNK: c-Jun $\mathrm{N}$-terminal kinase.

Table 2. Mouse models with Th17, Th2, and/or Th1 upregulation.

\begin{tabular}{|c|c|c|c|c|c|}
\hline Model & Mechanism & Features & Immune Profile & $\begin{array}{l}\text { Comparison to Human AD } \\
\text { (If Applicable) }\end{array}$ & References \\
\hline (11) Ikk2 $2^{\triangle N E S}$ & $\begin{array}{l}\text { Conditional } \\
\text { Ikk2-deficient mice that } \\
\text { do not express Ikk2 in } \\
\text { the dermis fibroblasts } \\
\text { of the face; develop } \\
\text { AD spontaneously. }\end{array}$ & $\begin{array}{c}\text { Keratinocyte } \\
\text { proliferation, mast } \\
\text { cell/eosinophilic } \\
\text { infiltrate, increased IgE. }\end{array}$ & $\begin{array}{l}\text { Th2 (IL-4, IL-5, IL-9, } \\
\text { IL-13, TSLP, and } \\
\text { Postn), Th17 (IL-17a) } \\
\text { IL-10/20 family of } \\
\text { genes (IL-10, IL-19, } \\
\text { IL-20, and IL-24) } \\
\text { No change in Th1 } \\
\text { or Th22 }\end{array}$ & $\begin{array}{c}\text { Unclear relevance of } \\
\text { pathogenesis; Ikk2-deficient } \\
\text { humans do not display } \\
\text { AD-like phenotype; the role of } \\
\text { fibroblasts in AD is not } \\
\text { characterized. } \\
\text { No barrier dysfunction; the } \\
\text { study reports an increase } \\
\text { in filaggrin. } \\
\text { The transcriptomic analysis } \\
\text { shows broad similarities with } \\
\text { human AD. }\end{array}$ & [3] \\
\hline $\begin{array}{l}\text { (12) MALT-1 } \\
\text { knockout }\end{array}$ & $\begin{array}{l}\text { MALT1 KO interferes } \\
\text { with TCR-induced gene } \\
\text { expression, lymphocyte } \\
\text { proliferation, and } \\
\text { regulatory T cell } \\
\text { development, leading } \\
\text { to Th2 expansion. }\end{array}$ & $\begin{array}{c}\text { Acanthosis, } \\
\text { hyperkeratosis, and } \\
\text { parakeratotic scaling, as } \\
\text { well as CD3+ T cell } \\
\text { infiltration. }\end{array}$ & $\begin{array}{l}\text { Th1 (IFN- } \gamma) \\
\text { Th2 (IL-4) } \\
\text { Th17 (IL-17) }\end{array}$ & $\begin{array}{l}\text { Dermatitis is reported in } \\
\text { humans with MALT1 } \\
\text { deficiency. (Demeyer } \\
\text { et al., 2019) }\end{array}$ & {$[22]$} \\
\hline $\begin{array}{l}\text { (13) Tmem79/ } \\
\text { Mattrin mutants }\end{array}$ & $\begin{array}{l}\text { No expression of the } \\
\text { protein mattrin; } \\
\text { Impaired lamellar } \\
\text { granular secretory } \\
\text { system, leading to } \\
\text { dysfunctional stratum } \\
\text { corneum. }\end{array}$ & $\begin{array}{l}\text { IL-17-dependent } \\
\text { acanthosis, } \\
\text { orthokeratosis, } \\
\text { inflammatory infiltrate. } \\
\text { Higher IgE response and } \\
\text { TEWL levels in ma/ma } \\
\text { after challenge with } \\
\text { house dust mite allergen } \\
\text { compared to } \\
\text { FLG }(\mathrm{ft} / \mathrm{ft}) \text { mice. }\end{array}$ & $\begin{array}{l}\text { Th1 (IFN- } \gamma) \\
\text { Th2 (IL-4) } \\
\text { Th17 (IL-17A) }\end{array}$ & $\begin{array}{c}\text { Matt gene mutation was found } \\
\text { to have only a small but } \\
\text { significant association in } \\
\text { human AD risk. }\end{array}$ & [23-26] \\
\hline $\begin{array}{l}\text { (14) } 2,4- \\
\text { dinitrofluoro- } \\
\text { benzene }\end{array}$ & $\begin{array}{l}\text { Optimized DNFB } \\
\text { dosing/scheduling to } \\
\text { induce AD. }\end{array}$ & $\begin{array}{l}\text { Lymphocytic and mast } \\
\text { cell infiltrate epidermal } \\
\text { hypertrophy and edema. }\end{array}$ & $\begin{array}{l}\text { Th1 (IFN- } \gamma) \\
\text { Th2 (IL-4) } \\
\text { Th17 (IL-17A) }\end{array}$ & $\begin{array}{l}\text { DNFB is also used to model } \\
\text { other proliferative } \\
\text { skin disorders. }\end{array}$ & [27] \\
\hline
\end{tabular}


Ten models noted Th22-related T-cell and cytokine changes in mice: Adam $17^{\mathrm{fl} / \mathrm{fl}} \mathrm{Sox} 9^{\mathrm{Cre}}$, NC/Nga, IL-23-induced, house dust mite (HDM)-induced, ovalbumin (OVA)-induced, chloromethylisothiazonilone or methylisothiazonilone (CMIT/MIT)-primed, oxazolone (OXA)-induced, flaky tail (ft), vitamin D3-induced, and K5-tTA-IL-22 mice (Table 3). These include all models whose transcriptomic homology with human AD have been evaluated: IL-23-induced (37\%), Adam $17^{\mathrm{fl} / \mathrm{fl}}$ Sox $9^{\mathrm{Cre}}(34 \%)$, NG/Nga (18\%), oxazolone-induced (17\%), ovalbumin-induced $(11 \%)$, and $f t(4 \%)$ mice [7]. On the other hand, Th22 activity was assessed in Ikk2 ${ }^{\Delta N E S}$ mice and found to be unchanged in affected animals (Table 2). All models with Th22 induction also exhibited Th17 upregulation, while six (models 17-18, 20, 22-24) also reported Th1 inflammation. Thus, among the models evaluated for this study, six murine models demonstrated broad upregulation of Th1, Th2, Th17, and Th22 inflammation: Adam $17^{\mathrm{fl} / \mathrm{fl}}$ Sox9 ${ }^{\mathrm{Cre}}$, IL-23-exposed, OXA-induced, OVA-induced, $f t$, and vitamin D3-induced mice.

Table 3. Th2 and/or Th1, Th17, or Th22 upregulation.

\begin{tabular}{|c|c|c|c|c|c|}
\hline Model & Mechanism & Features & Immune Profile & $\begin{array}{l}\text { Comparison to Human AD } \\
\text { (If Applicable) }\end{array}$ & References \\
\hline $\begin{array}{l}\text { (15) K5-tTA-IL-22 } \\
\text { mice }\end{array}$ & $\begin{array}{l}\text { Transgenic mice with } \\
\text { inducible expression } \\
\text { of IL-22 in the skin }\end{array}$ & $\begin{array}{c}\text { Thickening of the } \\
\text { epidermis and dermis, } \\
\text { spongiosis, } \\
\text { hyperkeratosis, } \\
\text { inflammatory cell } \\
\text { infiltration (eosinophils, } \\
\text { lymphocytes, } \\
\text { macrophages, } \\
\text { Langerhans cells, and } \\
\text { mast cells), and dermal } \\
\text { collagen accumulation }\end{array}$ & $\begin{array}{l}\text { Th2 (IL-4, IL-13) } \\
\text { Th17 (IL-17) } \\
\text { Th22 (IL-22) } \\
\text { Decreased IL-1 } \\
\text { (low IFN- } \gamma \text { ) }\end{array}$ & & [28] \\
\hline $\begin{array}{c}(16) \\
\mathrm{NC} / \mathrm{Nga}\end{array}$ & $\begin{array}{l}\text { Spontaneous AD } \\
\text { formation } \\
\text { (pathogenesis } \\
\text { undetermined) }\end{array}$ & $\begin{array}{c}\text { Moderate epidermal } \\
\text { hyperplasia with } \\
\text { elongation of rete ridges, } \\
\text { hyperkeratosis, increased } \\
\text { mast cells, and } \\
\text { eosinophils, increased IgE }\end{array}$ & $\begin{array}{c}\text { Th2 (IL-4, } \\
\text { IL-5)Th17/Th22 } \\
\text { (IL-17A, IL-22) }\end{array}$ & $\begin{array}{l}18 \% \text { homology with human } \\
\text { AD transcriptome }\end{array}$ & [29-31] \\
\hline $\begin{array}{c}\text { (17) IL-23 injection } \\
\text { in CCR2-deficient } \\
\text { mice }\end{array}$ & $\begin{array}{c}\text { IL-23 injection } \\
\text { stimulates } \\
\text { IL-22-dependent } \\
\text { dermal inflammation } \\
\text { and acanthosis; CCR2 } \\
\text { blockade shunts } \\
\text { immune response } \\
\text { toward Th2 and away } \\
\text { from Th1. }\end{array}$ & $\begin{array}{c}\text { Acanthosis, } \\
\text { hyperkeratosis, increased } \\
\text { epidermal thickness, } \\
\text { tissue eosinophilia. }\end{array}$ & $\begin{array}{c}\text { Th1 (IFN- } \gamma) \\
\text { Th2 (IL-13) } \\
\text { Th17/Th22 (IL-17A, } \\
\text { IL-22) }\end{array}$ & $\begin{array}{l}\text { 37\% homology with human } \\
\text { AD transcriptome }\end{array}$ & [32] \\
\hline $\begin{array}{l}\text { (18) Adam } 17^{\mathrm{fl} / \mathrm{fl}} \\
\text { Sox9 } 9^{\text {Cre }}\end{array}$ & $\begin{array}{l}\text { Adam17 deficiency in } \\
\text { Sox9-expressing tissue } \\
\text { causes dysbiosis, } \\
\text { leading to AD. }\end{array}$ & $\begin{array}{c}\text { Increased TEWL, } \\
\text { eczematous skin lesions, } \\
\text { increased IgE, } \\
\text { mononuclear infiltrate. } \\
\text { Dysbiosis with increased } \\
\text { colonization of S. aureus. }\end{array}$ & $\begin{array}{c}\text { Th1 } \\
\text { Th2 (CCL17) } \\
\text { Th17Th22 }\end{array}$ & $\begin{array}{l}\text { 34\% homology with human } \\
\text { AD transcriptome. } \\
\text { Adam17 deficiency in } \\
\text { humans leads to } \\
\text { AD-like phenotype. }\end{array}$ & {$[8,33]$} \\
\hline $\begin{array}{l}\text { (19) House dust } \\
\text { mite allergen } \\
\text { (HDM) }\end{array}$ & $\begin{array}{c}\text { Epicutaneous } \\
\text { sensitization to HDM }\end{array}$ & $\begin{array}{l}\text { Epidermal hyperplasia, } \\
\text { spongiosis, lymphocytic } \\
\text { infiltrate, elevated } \\
\text { serum IgE }\end{array}$ & $\begin{array}{c}\text { Th2 (IL-4, IL-5, IL-13)- } \\
\text { BALB/c and } \\
\text { C57BL/6 miceTh17 } \\
\text { (IL-17) } \\
\text { Th22 (IL-22)- } \\
\text { C57BL/6 mice }\end{array}$ & & {$[28,29]$} \\
\hline $\begin{array}{l}\text { (20) Ovalbumin } \\
\text { (OVA) with } \\
\text { mechanical barrier } \\
\text { disruption }\end{array}$ & $\begin{array}{c}\text { Tape-stripping } \\
\text { followed by } \\
\text { sensitization with } \\
\text { topical or } \\
\text { inhaled OVA. }\end{array}$ & $\begin{array}{l}\text { Epidermal and dermal } \\
\text { thickening with increased } \\
\text { collagen deposition, } \\
\text { infiltration of CD4+ T } \\
\text { cells, and eosinophils, } \\
\text { increased IgE. }\end{array}$ & $\begin{array}{c}\text { Th1 (IFN- } \gamma \text { ) } \\
\text { Th2 (IL-4, IL-5, IL-13), } \\
\text { Th17 (IL-17)- topical } \\
\text { OVA Th17 (IL-17)- } \\
\text { inhaled OVA } \\
\text { Th22 (IL-22) }\end{array}$ & $\begin{array}{l}\text { 11\% homology with human } \\
\text { AD transcriptome. }\end{array}$ & {$[29,34,35]$} \\
\hline
\end{tabular}


Table 3. Cont.

\begin{tabular}{|c|c|c|c|c|c|}
\hline Model & Mechanism & Features & Immune Profile & $\begin{array}{l}\text { Comparison to Human AD } \\
\text { (If Applicable) }\end{array}$ & References \\
\hline $\begin{array}{l}\text { (21) Chloromethyl- } \\
\text { isothiazonilone } \\
\text { (CMIT), } \\
\text { methylisothia- } \\
\text { zonilone (MIT) } \\
\text { and Ovalbumin }\end{array}$ & $\begin{array}{l}\text { CMIT/MIT with OVA } \\
\text { leads to a more } \\
\text { pronounced Th2 and } \\
\text { Th17 response than } \\
\text { OVA alone. }\end{array}$ & $\begin{array}{l}\text { Increased TEWL, } \\
\text { increased serum IgE, } \\
\text { mast cell infiltrate. }\end{array}$ & $\begin{array}{c}\text { Th2 (TSLP, IL-4, IL-6, } \\
\text { IL-13) } \\
\text { Th17 (IL-17A) }\end{array}$ & $\begin{array}{l}\text { Ability to differentially } \\
\text { enhance Th17 to replicate } \\
\text { certain endotypes. }\end{array}$ & {$[29,36]$} \\
\hline $\begin{array}{l}\text { (22) Spontaneous } \\
\text { recessive mouse } \\
\text { mutant flaky } \\
\text { tail (ft) }\end{array}$ & $\begin{array}{l}\text { Expression of } \\
\text { truncated profilaggrin } \\
\text { with functionally } \\
\text { absent filaggrin. }\end{array}$ & $\begin{array}{l}\text { Diffuse orthokeratotic } \\
\text { hyperkeratosis, } \\
\text { acanthosis, infiltrating } \\
\text { lymphocytes, eosinophils, } \\
\text { mononuclear cells, } \\
\text { increased TEWL. }\end{array}$ & $\begin{array}{c}\text { Th1 (IFN- } \gamma \text { ) } \\
\text { Th2 (IL-4, IL-5, IL-13), } \\
\text { Th17 (IL-17) upon } \\
\text { percutaneous } \\
\text { allergen exposure } \\
\text { with OVA } \\
\text { Th22 (IL-22) } \\
\text { Differences in } \\
\text { immune } \\
\text { upregulation } \\
\text { depending on mouse } \\
\text { strain: C57BL/6: Th1. } \\
\text { BALB/c: Th2/Th17 }\end{array}$ & $\begin{array}{l}\text { Filaggrin is the only } \\
\text { functionally characterized } \\
\text { gene in human } \mathrm{AD} \text {. } \\
4 \% \text { homology with human } \\
\text { AD transcriptome. }\end{array}$ & {$[29,37,38]$} \\
\hline $\begin{array}{l}\text { (23) Oxazolone } \\
\text { (OXA) }\end{array}$ & $\begin{array}{l}\text { Chronic exposure to } \\
\text { OXA (vs. allergic } \\
\text { contact dermatitis). }\end{array}$ & $\begin{array}{l}\text { Dermal infiltration of Th2 } \\
\text { lymphocytes, mast cells, } \\
\text { eosinophils, elevated IgE, } \\
\text { epidermal hyperplasia, } \\
\text { decreased expression of } \\
\text { filaggrin, loricrin, and } \\
\text { involucrin. Decreased } \\
\text { stratum corneum } \\
\text { ceramide content, } \\
\text { decreased stratum } \\
\text { corneum hydration, } \\
\text { transepidermal water } \\
\text { loss, and impaired } \\
\text { lamellar body secretion. }\end{array}$ & $\begin{array}{l}\text { Th1 (IFN- } \gamma \text { ) } \\
\text { Th2 (IL-4, IL-13) } \\
\text { Th17 (IL-17) } \\
\text { Th22 (IL-22) }\end{array}$ & $\begin{array}{l}\text { 17\% homology with human } \\
\text { AD transcriptome. }\end{array}$ & $\begin{array}{c}{[29,34,39,} \\
40]\end{array}$ \\
\hline $\begin{array}{l}\text { (24) Vitamin D3 } \\
\text { administration }\end{array}$ & $\begin{array}{l}\text { Vitamin D3 or its } \\
\text { analog MC903 } \\
\text { (calcipotriol) induces } \\
\text { overexpression } \\
\text { of TSLP }\end{array}$ & $\begin{array}{l}\text { Epidermal hyperplasia, } \\
\text { dermal inflammatory } \\
\text { infiltrate of eosinophils, } \\
\text { CD3, CD4, CD11c, } \\
\text { mast cells }\end{array}$ & $\begin{array}{l}\text { Th1/Th2 mixed } \\
\text { (TSLP, IL-4, IL-5, } \\
\text { IL-13, IL-31, IL-10, } \\
\text { IL-8, IFN- } \gamma, \text { TNF) } \\
\text { Th17 (IL-17) } \\
\text { Th22 (IL-22) }\end{array}$ & & {$[29,41]$} \\
\hline
\end{tabular}

Three models are notable for outlining methods that may aid in selectively modulating inflammation: OVA with CMIT/MIT exposure, $f t$ mice, and HDM-induced mice [28,36-38]. While ovalbumin commonly to induce eczema in mice, Go et al. (2020), found that mice sensitized with CMIT/MIT before OVA displayed an augmented Th17 reaction than mice exposed to OVA alone [36]. Likewise, Fallon et al. (2009) demonstrated higher Th17 activation in BALB/c mice harboring the $(f t)$ mutation compared to C57BL/ 6 mice [37]. Similarly, among models that demonstrate Th22 upregulation, the HDM-induced model allows for selective suppression of Th22 response with the use of BALB/c instead of C57BL/ 6 mice [28]. Although these methods demonstrate the potential for modeling multiple endotypes within a single genetic strain, both OVA-induced and $f t$ mice share the least homology with the human AD transcriptome, at $11 \%$ and $4 \%$, respectively [7], while the transcriptomic homology of the HDM-induced model has not been evaluated.

Existing drug validation studies that evaluated the effects of FDA-approved and investigational therapies in select models provide insight into their translational utility (Table 4). Corticosteroids tested against NC/Nga mice and OXA-challenged mice led to improvements in histopathologic features of $\mathrm{AD}$, while also reducing the expression of Th2 cytokines in NC/Nga mice and Th2/Th17-related cytokines in OXA-challenged mice [42]. Calcineurin inhibitors tacrolimus and pimecrolimus have been tested widely against NC/Nga, OXA-challenged, Ikk2 $\triangle^{\text {NES }}$, DNFB-challenged, and HDM-induced mice [3,43,44], 
with models demonstrating variable response to the indicated compounds in terms of histopathologic improvements. While treatment with tacrolimus and pimecrolimus led to reductions in Th2 and Th17 activity, inflammatory cytokine suppression was not evaluated in all tested models. Conversely, 2,4-dinitrofluoro-benzene (DNFB)-challenged and HDMinduced mice showed minimal inflammatory improvement with cyclosporine treatment, with the former demonstrating partial suppression of IL-13 and TNF- $\alpha$ upregulation [45]. Crisaborole and Compd3, which act via PDE4 inhibition, demonstrated efficacy against calcipotriol-induced AD lesions, demonstrating reductions in TSLP expression and skin swelling [46,47]. Novel Janus kinase (JAK) inhibitors have also been studied broadly in numerous models, including NC/Nga, Ikk2 $\Delta^{\mathrm{NES}}$, DNFB-challenged, HDM-induced, and human skin-grafted mice $[44,45,48,49]$. Mice treated with JAK inhibitors delgocitinib and tofacitnib led to broad inhibition of Th2-related cytokines, as well as improvements in clinical severity and barrier function $[44,45,48,49]$.

Table 4. The effect of select FDA-approved or investigational agents on specific models.

\begin{tabular}{|c|c|c|c|c|}
\hline Model & Therapeutic Agent & Class & Effects on Mice & Reference \\
\hline $\mathrm{NC} / \mathrm{Nga}$ & Dexamethasone & Corticosteroid & $\begin{array}{l}\text { Reduction of Th2- (IL-4, IL-5) and } \\
\text { Th17-related (IL-17A) cytokines. } \\
\text { Reduction in tissue swelling and } \\
\text { immune cell infiltration. }\end{array}$ & [42] \\
\hline $\mathrm{NC} / \mathrm{Nga}$ & Delgocitinib (JTE-052) & JAK inhibitor & $\begin{array}{l}\text { Improved clinical score, } \\
\text { decreased TEWL, restoration of } \\
\text { hygroscopic amino acids needed } \\
\text { for stratum corneum hydration }\end{array}$ & [48] \\
\hline $\mathrm{NC} / \mathrm{Nga}$ & Tacrolimus & Calcineurin inhibitor & $\begin{array}{l}\text { Reduction of Th1- (IFN- } \gamma) \text {, Th2- } \\
\text { (IL-5, IL-13), Th17-related } \\
\text { (IL-17) cytokines }\end{array}$ & [44] \\
\hline MC903 (calcipotriol) & Crisaborole & PDE4 inhibitor & $\begin{array}{l}\text { Reduction in ear thickness and } \\
\text { skin swelling. }\end{array}$ & [46] \\
\hline MC903 (calcipotriol) & Compd3 & Novel PDE4 inhibitor & Reduction in TSLP expression & [47] \\
\hline Oxazolone-challenged & $\begin{array}{l}\text { Pimecrolimus } \\
\text { Methylprednisolone }\end{array}$ & $\begin{array}{l}\text { Calcineurin inhibitor } \\
\text { Corticosteroid }\end{array}$ & $\begin{array}{l}\text { Decrease in TEWL and increased } \\
\text { stratum corneum hydration } \\
\text { Reduced expression of IL- } 1 \alpha \text {, } \\
\text { TNF- } \alpha, \text { PAR-2, and TSLP }\end{array}$ & [43] \\
\hline Ikk $2^{\Delta N E S}$ & $\begin{array}{l}\text { Tacrolimus } \\
\text { Tofacitinib } \\
\quad \text { Stattic }\end{array}$ & $\begin{array}{l}\text { Calcineurin inhibitor } \\
\text { JAK inhibitor } \\
\text { Stat3 inhibitor }\end{array}$ & $\begin{array}{l}\text { Partial decrease in the infiltration } \\
\text { of leukocytes and eosinophils; } \\
\text { partial decrease in } \\
\text { epidermal swelling. }\end{array}$ & [3] \\
\hline DNFB-challenged & Cyclosporine & Calcineurin inhibitor & $\begin{array}{l}\text { Partial suppression of IL-13 and } \\
\text { TNF- } \alpha \text { upregulation. No effect on } \\
\text { inflammatory changes. }\end{array}$ & [45] \\
\hline DNFB-challenged & Delgocitinib (JTE-052) & JAK inhibitor & $\begin{array}{l}\text { Reduction in IL-4, IL-13, and } \\
\text { TNF- } \alpha \text { expression. Reduction in } \\
\text { acanthosis, spongiosis, and } \\
\text { inflammatory infiltrate. }\end{array}$ & [45] \\
\hline House dust mite allergen & Cyclosporine & Calcineurin inhibitor & No effect on ear thickness & [45] \\
\hline House dust mite allergen & Delgocitinib (JTE-052) & JAK inhibitor & $\begin{array}{l}\text { Reduction in ear thickness with } \\
\text { greater efficacy than cyclosporine. }\end{array}$ & [45] \\
\hline House dust mite allergen & Tofacitinib & JAK inhibitor & $\begin{array}{c}\text { Diminished IL-1 } \beta, \text { TNF- } \alpha \text {, TSLP, } \\
\text { IL-4, IL-13 }\end{array}$ & [49] \\
\hline Human skin graft model & Delgocitinib (JTE-052) & JAK inhibitor & Increased FLG protein expression & [48] \\
\hline
\end{tabular}




\section{Discussion}

Ongoing efforts to demystify the complexity of AD have led to the identification of immunologically distinct phenotypes that vary based on permutations of Th1, Th17, and Th22 inflammation [1]. AD in Asian patients is thought to harbor heightened Th17/Th22 bias, AD in African Americans have Th2/Th22 skewing with Th17 attenuation, and European American lesions exhibit Th1/Th17 suppression [50]. Similarly, pediatric AD is notable for an unaltered Th1 profile against a background of Th17/Th22 upregulation [51], while adult $\mathrm{AD}$ is characterized by broad inflammatory induction of all axes [52]. Studies have also observed immune heterogeneity across adult subpopulations when stratified by age group, noting more robust Th22 upregulation in younger adults compared to older adults [52]. The potential to tailor treatments based on a patient's unique endotype represents an exciting new frontier in clinical drug discovery as the treatment paradigm for AD shifts from systemic interventions to targeted therapies [52].

Thus, the ability to reproduce these endotypes in mice represents a critical step toward optimizing the selection of translationally relevant mouse models, particularly in an era driven by the rising importance of personalized medicine [52]. Kim et al. (2019) previously outlined several features that warrant consideration in the selection of a mouse model, including gross phenotype/histology, serum profile, transcriptomic similarities, and immunophenotype [6]. More recently, Gilhar et al. (2020) proposed a list of criteria that animal models of human AD should meet, which included the consideration of inflammatory patterns [29]. An in-depth assessment of a model's unique immunological signature and the ability to individually modulate specific immune axes may be key for future preclinical studies seeking to assess how novel biologics blockade the inflammatory upregulation that defines specific $\mathrm{AD}$ endotypes, as selection based on genetics, phenotype, or gene expression profile may not adequately capture the immunologic complexity of AD. For instance, while Ikk ${ }^{\Delta \mathrm{NES}}$ mice are notable for their transcriptomic homology with human $\mathrm{AD}$, their lack of Th22 induction potentially limits their utility for the study of Asian, pediatric, and young adult endotypes that are notable for Th22 inflammation [3]. Similarly, NC/Nga mice characterized by high transcriptomic homology with the human $\mathrm{AD}$ transcriptome may be of limited translational relevance for the European American endotype given its marked Th17 induction $[30,31,50]$.

Looking to the future, newer techniques such as the bioengineered humanized skin model [17] and the transplantation of stimulated peripheral blood mononuclear cells (PBMC) [29] hold great promise in their potential ability reproduce population-specific immune signatures in mice. While these techniques are further explored and characterized, a more practical approach to improving existing models' translational relevance can be guided by efforts to increase their versatility by exploring modifications that selectively modulate inflammatory responses, such as the use of CMIT/MIT prior to OVA sensitization to bolster Th17 response [36], or by characterizing the inflammatory response demonstrated by different mouse strains to aid in the selection of a model that most closely mimics a desired immune signature [28,37]. For instance, researchers seeking to model the European American AD endotype, noted for its Th17 attenuation, may use C57BL/ 6 mice instead of $\mathrm{BALB} / \mathrm{c}$ mice within the $\mathrm{ft}$ model in order to abrogate Th17 response [37]. Similarly, the Asian or pediatric endotypes may be best reproduced with HDM-induced C57BL/ 6 mice to bolster Th22 inflammation [37].

In both cases, a concern lies in the task of capturing the complex features of $\mathrm{AD}$ in their entirety, which is further complicated by the lack of consensus regarding the features that define such criteria [29]. Thus, an additional measure to optimize the utility of existing models may involve validating therapies across a heterogenous panel of animal models, taking advantage of the common mediators that underlie cutaneous inflammation in different models. Defining an agent's pattern of anti-inflammatory activity across multiple models that encompass all relevant features of $\mathrm{AD}$ may overcome their individual limitations and provide a broader picture of therapeutic response. 
There are several additional limitations to selecting a model based on its endotype. Many models have not been completely evaluated for their immunologic signatures, while existing drug validation studies provide only limited insight into translational utility of tested models given their incomplete profiling of inflammatory suppression. Further studies are warranted to detail the inflammatory changes in all AD mouse models, and to characterize the degree of similarity in therapeutic response between murine AD and human AD. Moreover, generalizability is bound by interspecies differences in immune systems and their relationship to relevant biological pathways [6,53]. For instance, IL-17 is mostly produced by Th17 cells in humans and by $\gamma \delta \mathrm{T}$ cells in mice. While the contribution of IL-17-producing $\gamma \delta \mathrm{T}$ cells has been assessed in human psoriasis, their role in human AD remains uncharacterized [53,54]. Similarly, although humanized skin and PBMC models represent promising strategies to recreate specific inflammatory signatures in mice, xenografts are still subject to interference caused by interactions with host physiology [17]

In conclusion, as our understanding of the specific roles of the inflammatory axes crystallizes, future studies may shift toward optimizing the efficacy of targeted therapies based on their specific effects on the immune factors that determine specific AD phenotypes. Thus, in an effort to overcome the challenge of selecting a mouse model that broadly captures the intricacy of human $\mathrm{AD}$, immunophenotypic considerations should play a more central role in the selection of AD mouse models in future preclinical studies.

Author Contributions: Conceptualization, J.C. and S.G.K.; methodology, J.C.; software, J.C.; validation, J.C., S.G.K. and M.M.K.; formal analysis, J.C.; investigation, J.C.; resources, J.C.; data curation, J.C.; writing—original draft preparation, J.C., N.S., Y.S.R., Z.B.; writing-review and editing, J.C., N.S., Y.S.R., Z.B., M.P.A.; visualization, J.C..; supervision, S.G.K., M.M.K.; project administration, J.C., S.G.K.; funding acquisition: not applicable. All authors have read and agreed to the published version of the manuscript.

Funding: This research received no external funding.

Institutional Review Board Statement: Not applicable.

Informed Consent Statement: Not applicable.

Conflicts of Interest: Shawn G. Kwatra is an advisory board member/consultant for Abbvie, Galderma, Incyte Corporation, Pfizer Inc., Regeneron Pharmaceuticals, and Kiniksa Pharmaceuticals and has received grant funding from Galderma, Pfizer Inc. and Kiniksa Pharmaceuticals. The authors declare no conflict of interest.

\section{References}

1. Eyerich, K.; Novak, N. Immunology of atopic eczema: Overcoming the Th1/Th2 paradigm. Allergy 2013, 68, 974-982. [CrossRef]

2. Ma, C.A.; Stinson, J.R.; Zhang, Y.; Abbott, J.K.; A Weinreich, M.; Hauk, P.J.; Reynolds, P.R.; Lyons, J.J.; Nelson, C.G.; Ruffo, E.; et al. Germline hypomorphic CARD11 mutations in severe atopic disease. Nat. Genet. 2017, 49, 1192-1201, Correction in 2017, $49,1661$. [CrossRef]

3. Nunomura, S.; Ejiri, N.; Kitajima, M.; Nanri, Y.; Arima, K.; Mitamura, Y.; Yoshihara, T.; Fujii, K.; Takao, K.; Imura, J.; et al. Establishment of a Mouse Model of Atopic Dermatitis by Deleting Ikk2 in Dermal Fibroblasts. J. Investig. Dermatol. 2019, 139, 1274-1283. [CrossRef]

4. Scharschmidt, T.C.; Segre, J.A. Modeling Atopic Dermatitis with Increasingly Complex Mouse Models. J. Investig. Dermatol. 2008, 128, 1061-1064. [CrossRef]

5. Gutermuth, J.; Ollert, M.; Ring, J.; Behrendt, H.; Jakob, T. Mouse Models of Atopic Eczema Critically Evaluated. Int. Arch. Allergy Immunol. 2004, 135, 262-276. [CrossRef]

6. Kim, D.Y.; Kobayashi, T.; Nagao, K. Research Techniques Made Simple: Mouse Models of Atopic Dermatitis. J. Investig. Dermatol. 2019, 139, 984-990.e1. [CrossRef] [PubMed]

7. Ewald, D.A.; Noda, S.; Oliva, M.; Litman, T.; Nakajima, S.; Li, X.; Xu, H.; Workman, C.T.; Scheipers, P.; Svitacheva, N.; et al. Major differences between human atopic dermatitis and murine models, as determined by using global transcriptomic profiling. J. Allergy Clin. Immunol. 2017, 139, 562-571. [CrossRef] [PubMed]

8. Woodring, T.; Kobayashi, T.; Kim, D.Y.; Nagao, K. ADAM17-Deficient Mice Model the Transcriptional Signature of Human Atopic Dermatitis. J. Investig. Dermatol. 2018, 138, 2283-2286. [CrossRef] [PubMed]

9. Kim, B.E.; Leung, D.Y.; Boguniewicz, M.; Howell, M.D. Loricrin and involucrin expression is down-regulated by Th2 cytokines through STAT-6. Clin. Immunol. 2008, 126, 332-337. [CrossRef] [PubMed] 
10. Sehra, S.; Bruns, H.A.; Ahyi, A.-N.N.; Nguyen, E.T.; Schmidt, N.W.; Michels, E.G.; Von Bülow, G.-U.; Kaplan, M.H. IL-4 Is a Critical Determinant in the Generation of Allergic Inflammation Initiated by a Constitutively Active Stat6. J. Immunol. 2008, 180, 3551-3559. [CrossRef]

11. Sehra, S.; Krishnamurthy, P.; Koh, B.; Zhou, H.-M.; Seymour, L.; Akhtar, N.; Travers, J.B.; Turner, M.J.; Kaplan, M.H. Increased Th2 activity and diminished skin barrier function cooperate in allergic skin inflammation. Eur. J. Immunol. 2016, 46, 2609-2613. [CrossRef] [PubMed]

12. Zheng, T.; Oh, M.H.; Oh, S.Y.; Schroeder, J.T.; Glick, A.B.; Zhu, Z. Transgenic Expression of Interleukin-13 in the Skin Induces a Pruritic Dermatitis and Skin Remodeling. J. Investig. Dermatol. 2009, 129, 742-751. [CrossRef] [PubMed]

13. Yoo, J.; Omori, M.; Gyarmati, D.; Zhou, B.; Aye, T.; Brewer, A.; Comeau, M.R.; Campbell, D.J.; Ziegler, S.F. Spontaneous atopic dermatitis in mice expressing an inducible thymic stromal lymphopoietin transgene specifically in the skin. J. Exp. Med. 2005, 202, 541-549. [CrossRef]

14. Imai, Y.; Yasuda, K.; Sakaguchi, Y.; Haneda, T.; Mizutani, H.; Yoshimoto, T.; Nakanishi, K.; Yamanishi, K. Skin-specific expression of IL-33 activates group 2 innate lymphoid cells and elicits atopic dermatitis-like inflammation in mice. Proc. Natl. Acad. Sci. USA 2013, 110, 13921-13926. [CrossRef]

15. Altin, J.A.; Tian, L.; Liston, A.; Bertram, E.M.; Goodnow, C.C.; Cook, M.C. Decreased T-cell receptor signaling through CARD11 differentially compromises forkhead box protein 3-positive regulatory versus TH2 effector cells to cause allergy. J. Allergy Clin. Immunol. 2011, 127, 1277-1285.e5. [CrossRef]

16. Jun, J.E.; Wilson, L.E.; Vinuesa, C.G.; Lesage, S.; Blery, M.; Miosge, L.A.; Cook, M.C.; Kucharska, E.M.; Hara, H.; Penninger, J.M.; et al. Identifying the MAGUK Protein Carma-1 as a Central Regulator of Humoral Immune Responses and Atopy by Genome-Wide Mouse Mutagenesis. Immunity 2003, 18, 751-762. [CrossRef]

17. Guerrero-Aspizua, S.; Carretero, M.; Conti, C.J.; Del Río, M. The importance of immunity in the development of reliable animal models for psoriasis and atopic dermatitis. Immunol. Cell Biol. 2020, 98, 626-638. [CrossRef] [PubMed]

18. Carretero, M.; Guerrero-Aspizua, S.; Illera, N.; Galvez, V.; Navarro, M.; García-García, F.; Dopazo, J.; Jorcano, J.L.; Larcher, F.; Del Río, M. Differential Features between Chronic Skin Inflammatory Diseases Revealed in Skin-Humanized Psoriasis and Atopic Dermatitis Mouse Models. J. Investig. Dermatol. 2016, 136, 136-145. [CrossRef] [PubMed]

19. Fujii, M.; Ohgami, S.; Asano, E.; Nakayama, T.; Toda, T.; Nabe, T.; Ohya, S. Brain allopregnanolone induces marked scratching behaviour in diet-induced atopic dermatitis mouse model. Sci. Rep. 2019, 9, 2364. [CrossRef] [PubMed]

20. Dillon, S.R.; Sprecher, C.; Hammond, A.; Bilsborough, J.; Rosenfeld-Franklin, M.; Presnell, S.R.; Haugen, H.S.; Maurer, M.; Harder, B.; Johnston, J.; et al. Interleukin 31, a cytokine produced by activated T cells, induces dermatitis in mice. Nat. Immunol. 2004, 5, 752-760. [CrossRef]

21. Yasuda, T.; Fukada, T.; Nishida, K.; Nakayama, M.; Matsuda, M.; Miura, I.; Dainichi, T.; Fukuda, S.; Kabashima, K.; Nakaoka, S.; et al. Hyperactivation of JAK1 tyrosine kinase induces stepwise, progressive pruritic dermatitis. J. Clin. Investig. 2016, 126, 2064-2076. [CrossRef]

22. Demeyer, A.; Van Nuffel, E.; Baudelet, G.; Driege, Y.; Kreike, M.; Muyllaert, D.; Staal, J.; Beyaert, R. MALT1-Deficient Mice Develop Atopic-Like Dermatitis Upon Aging. Front. Immunol. 2019, 10, 2330. [CrossRef] [PubMed]

23. Emrick, J.; Mathur, A.; Wei, J.; Gracheva, E.O.; Gronert, K.; Rosenblum, M.D.; Julius, D. Tissue-specific contributions of Tmem79 to atopic dermatitis and mast cell-mediated histaminergic itch. Proc. Natl. Acad. Sci. USA 2018, 115, E12091-E12100. [CrossRef] [PubMed]

24. Sasaki, T.; Shiohama, A.; Kubo, A.; Kawasaki, H.; Ishida-Yamamoto, A.; Yamada, T.; Hachiya, T.; Shimizu, A.; Okano, H.; Kudoh, J.; et al. A homozygous nonsense mutation in the gene for Tmem79, a component for the lamellar granule secretory system, produces spontaneous eczema in an experimental model of atopic dermatitis. J. Allergy Clin. Immunol. 2013, 132, 1111-1120.e4. [CrossRef] [PubMed]

25. Saunders, S.P.; Goh, C.S.; Brown, S.J.; Palmer, C.N.; Porter, R.M.; Cole, C.; Campbell, L.E.; Gierlinski, M.; Barton, G.J.; Schneider, G.; et al. Tmem79/Matt is the matted mouse gene and is a predisposing gene for atopic dermatitis in human subjects. J. Allergy Clin. Immunol. 2013, 132, 1121-1129. [CrossRef]

26. Saunders, S.P.; Floudas, A.; Moran, T.; Byrne, C.M.; Rooney, M.D.; Fahy, C.M.R.; Geoghegan, J.A.; Iwakura, Y.; Fallon, P.G.; Schwartz, C. Dysregulated skin barrier function in Tmem79 mutant mice promotes IL-17A-dependent spontaneous skin and lung inflammation. Allergy 2020, 75, 3216-3227. [CrossRef]

27. Kitamura, A.; Takata, R.; Aizawa, S.; Watanabe, H.; Wada, T. A murine model of atopic dermatitis can be generated by painting the dorsal skin with hapten twice 14 days apart. Sci. Rep. 2018, 8, 5988. [CrossRef] [PubMed]

28. Lou, H.; Lu, J.; Choi, E.B.; Oh, M.H.; Jeong, M.; Barmettler, S.; Zhu, Z.; Zheng, T. Expression of IL-22 in the Skin Causes Th2-Biased Immunity, Epidermal Barrier Dysfunction, and Pruritus via Stimulating Epithelial Th2 Cytokines and the GRP Pathway. J. Immunol. 2017, 198, 2543-2555. [CrossRef] [PubMed]

29. Gilhar, A.; Reich, K.; Keren, A.; Kabashima, K.; Steinhoff, M.; Paus, R. Mouse models of atopic dermatitis: A critical reappraisal. Exp. Dermatol. 2020, 10. [CrossRef]

30. Matsuda, H.; Watanabe, N.; Geba, G.P.; Sperl, J.; Tsudzuki, M.; Hiroi, J.; Matsumoto, M.; Ushio, H.; Saito, S.; Askenase, P.W.; et al. Development of atopic dermatitis-like skin lesion with IgE hyperproduction in NC/Nga mice. Int. Immunol. 1997, 9, 461-466. [CrossRef] 
31. Kim, S.H.; Seong, G.S.; Choung, S.-Y. Fermented Morinda citrifolia (Noni) Alleviates DNCB-Induced Atopic Dermatitis in NC/Nga Mice through Modulating Immune Balance and Skin Barrier Function. Nutrients 2020, 12, 249. [CrossRef] [PubMed]

32. Bromley, S.K.; Larson, R.P.; Ziegler, S.F.; Luster, A.D. IL-23 Induces Atopic Dermatitis-Like Inflammation Instead of Psoriasis-Like Inflammation in CCR2-Deficient Mice. PLOS ONE 2013, 8, e58196. [CrossRef] [PubMed]

33. Kobayashi, T.; Glatz, M.; Horiuchi, K.; Kawasaki, H.; Akiyama, H.; Kaplan, D.H.; Kong, H.H.; Amagai, M.; Nagao, K. Dysbiosis and Staphylococcus aureus Colonization Drives Inflammation in Atopic Dermatitis. Immunity 2015, 42, 756-766. [CrossRef] [PubMed]

34. Jin, H.; He, R.; Oyoshi, M.; Geha, R.S. Animal models of atopic dermatitis. J. Invest. Dermatol. 2009, 129, 31-40. [CrossRef] [PubMed]

35. Wang, G.; Savinko, T.; Wolff, H.; Dieu-Nosjean, M.C.; Kemény, L.; Homey, B.; Lauerma, A.I.; Alenius, H. Repeated epicutaneous exposures to ovalbumin progressively induce atopic dermatitis-like skin lesions in mice. Clin. Exp. Allergy 2006, 37, 151-161. [CrossRef]

36. Go, H.-N.; Lee, S.-H.; Cho, H.-J.; Ahn, J.-R.; Kang, M.-J.; Lee, S.-Y.; Kim, H.-J. Effects of chloromethylisothiazolinone/ methylisothiazolinone (CMIT/MIT) on Th2/Th17-related immune modulation in an atopic dermatitis mouse model. Sci. Rep. 2020, 10, 1-9. [CrossRef]

37. Fallon, P.G.; Sasaki, T.; Sandilands, A.; Campbell, L.E.; Saunders, S.P.; Mangan, N.E.; Callanan, J.J.; Kawasaki, H.; Shiohama, A.; Kubo, A.; et al. A homozygous frameshift mutation in the mouse Flg gene facilitates enhanced percutaneous allergen priming. Nat. Genet. 2009, 41, 602-608. [CrossRef]

38. Oyoshi, M.K.; Murphy, G.F.; Geha, R. Filaggrin-deficient mice exhibit TH17-dominated skin inflammation and permissiveness to epicutaneous sensitization with protein antigen. J. Allergy Clin. Immunol. 2009, 124, 485-493. [CrossRef]

39. Heo, W.I.; Lee, K.E.; Hong, J.Y.; Kim, M.N.; Oh, M.S.; Kim, Y.S.; Kim, K.W.; Kim, K.E.; Sohn, M.H. The role of interleukin-17 in mouse models of atopic dermatitis and contact dermatitis. Clin. Exp. Dermatol. 2015, 40, 665-671. [CrossRef]

40. Man, M.-Q.; Hatano, Y.; Lee, S.H.; Man, M.; Chang, S.; Feingold, K.R.; Leung, D.Y.; Holleran, W.M.; Uchida, Y.; Elias, P.M. Characterization of a Hapten-Induced, Murine Model with Multiple Features of Atopic Dermatitis: Structural, Immunologic, and Biochemical Changes following Single Versus Multiple Oxazolone Challenges. J. Investig. Dermatol. 2008, 128, 79-86. [CrossRef]

41. Moosbrugger-Martinz, V.; Schmuth, M.; Dubrac, S. A Mouse Model for Atopic Dermatitis Using Topical Application of Vitamin D3 or of Its Analog MC903. Methods Mol. Biol. 2017, 1559, 91-106. [CrossRef]

42. Lee, T.-Y.; Kim, D.-J.; Won, J.-N.; Lee, I.-H.; Sung, M.-H.; Poo, T.-H.K.A.H. Oral Administration of Poly- $\gamma$-Glutamate Ameliorates Atopic Dermatitis in Nc/Nga Mice by Suppressing Th2-Biased Immune Response and Production of IL-17A. J. Investig. Dermatol. 2014, 134, 704-711. [CrossRef] [PubMed]

43. Yoon, N.Y.; Jung, M.Y.; Kim, D.H.; Lee, H.J.; Choi, E.H. Topical glucocorticoid or pimecrolimus treatment suppresses thymic stromal lymphopoietin-related allergic inflammatory mechanism in an oxazolone-induced atopic dermatitis murine model. Arch. Dermatol. Res. 2015, 307, 569-581. [CrossRef]

44. Natsume, C.; Aoki, N.; Aoyama, T.; Senda, K.; Matsui, M.; Ikegami, A.; Tanaka, K.; Azuma, Y.-T.; Fujita, T. Fucoxanthin Ameliorates Atopic Dermatitis Symptoms by Regulating Keratinocytes and Regulatory Innate Lymphoid Cells. Int. J. Mol. Sci. 2020, 21, 2180. [CrossRef] [PubMed]

45. Tanimoto, A.; Shinozaki, Y.; Yamamoto, Y.; Katsuda, Y.; Taniai-Riya, E.; Toyoda, K.; Kakimoto, K.; Kimoto, Y.; Amano, W.; Konishi, N.; et al. A novel JAK inhibitor JTE-052 reduces skin inflammation and ameliorates chronic dermatitis in rodent models: Comparison with conventional therapeutic agents. Exp. Dermatol. 2017, 27, 22-29. [CrossRef] [PubMed]

46. Chu, Z.; Xu, Q.; Zhu, Q.; Ma, X.; Mo, J.; Lin, G.; Zhao, Y.; Gu, Y.; Bian, L.; Shao, L.; et al. Design, synthesis and biological evaluation of novel benzoxaborole derivatives as potent PDE4 inhibitors for topical treatment of atopic dermatitis. Eur. J. Med. Chem. 2021, 213, 113171. [CrossRef] [PubMed]

47. Dong, C.; Virtucio, C.; Zemska, O.; Baltazar, G.; Zhou, Y.; Baia, D.; Jones-Iatauro, S.; Sexton, H.; Martin, S.; Dee, J.; et al. Treatment of skin inflammation with benzoxaborole PDE inhibitors: Selectivity, cellular activity, and effect on cytokines associated with skin inflammation and skin architecture changes. J. Pharmacol. Exp. Ther. 2016, 358, 413-422. [CrossRef]

48. Amano, W.; Nakajima, S.; Kunugi, H.; Numata, Y.; Kitoh, A.; Egawa, G.; Dainichi, T.; Honda, T.; Otsuka, A.; Kimoto, Y.; et al. The Janus kinase inhibitor JTE-052 improves skin barrier function through suppressing signal transducer and activator of transcription 3 signaling. J. Allergy Clin. Immunol. 2015, 136, 667-677.e7. [CrossRef]

49. Fukuyama, T.; Ehling, S.; Wilzopolski, J.; Bäumer, W. Comparison of topical tofacitinib and $0.1 \%$ hypochlorous acid in a murine atopic dermatitis model. BMC Pharmacol. Toxicol. 2018, 19, 37. [CrossRef] [PubMed]

50. Sanyal, R.D.; Pavel, A.B.; Glickman, J.; Chan, T.C.; Zheng, X.; Zhang, N.; Cueto, I.; Peng, X.; Estrada, Y.; Fuentes-Duculan, J.; et al. Atopic dermatitis in African American patients is TH2/TH22-skewed with TH1/TH17 attenuation. Ann. Allergy Asthma Immunol. 2019, 122, 99-110.e6. [CrossRef] [PubMed]

51. Brunner, P.M.; Israel, A.; Leonard, A.; Pavel, A.B.; Kim, H.J.; Zhang, N.; Czarnowicki, T.; Patel, K.; Murphrey, M.; Ramsey, K.; et al. Distinct transcriptomic profiles of early-onset atopic dermatitis in blood and skin of pediatric patients. Ann. Allergy Asthma Immunol. 2019, 122, 318-330.e3. [CrossRef] [PubMed]

52. Nomura, T.; Wu, J.; Kabashima, K.; Guttman-Yassky, E. Endophenotypic Variations of Atopic Dermatitis by Age, Race, and Ethnicity. J. Allergy Clin. Immunol. Pr. 2020, 8, 1840-1852. [CrossRef] [PubMed] 
53. Nakajima, S.; Nomura, T.; Common, J.E.; Kabashima, K. Insights into atopic dermatitis gained from genetically defined mouse models. J. Allergy Clin. Immunol. 2019, 143, 13-25. [CrossRef]

54. Cai, Y.; Shen, X.; Ding, C.; Qi, C.; Li, K.; Li, X.; Jala, V.R.; Zhang, H.-G.; Wang, T.; Zheng, J.; et al. Pivotal Role of Dermal IL-17-Producing $\gamma \delta \mathrm{T}$ Cells in Skin Inflammation. Immunity 2011, 35, 596-610. [CrossRef] 